\title{
The Identification of Two Antibacterial Products of the Marine Planktonic Alga Isochrysis galbana
}

\author{
By D. L. BRUCE AND D. C. B. DUFF \\ Department of Microbiology, University of B.C., \\ Vancouver, British Columbia \\ AND N. J. ANTIA \\ Fisheries Research Board of Canada, Vancouver Laboratory, \\ Vancouver, British Columbia \\ (Accepted for publication I8 March 1967) \\ SUMMARY \\ The acetone-extractable antibacterial components of Isochrysis galbana \\ have been resolved by paper-chromatography and bioautography. Two \\ pigmented products with similar antibacterial properties were obtained which \\ were tentatively identified from their absorption spectra as chlorophyll $a$ de- \\ rivatives, probably pheophytin $a$ and an atypical chlorophyllide $a$.
}

\section{INTRODUCTION}

A survey of marine planktonic algae for antibacterial activity (Duff, Bruce \& Antia, 1966) revealed a wide range and degree of activity in several algal species, of which a diatom Skeletonema costatum and the chrysophyte Isochrysis galbana appeared most promising for further examination. The study of $I$. galbana lead to the characterization of the products responsible for the observed activity. The resolution of acetone extracts of this alga into two active components and their identification as derivatives of chlorophyll $a$ are reported in this paper.

\section{MET HODS}

Algal culture and extracts. Isochrysis galbana Parke was grown in axenic culture and the algal material was harvested and dried as described by Antia \& Kalmakoff (1965).

Algal extracts were prepared by three methods. Method $(a)$ involved sequential extractions with three solvent systems (acetone; chloroform; chloroform + methanol $(\mathrm{I}+\mathrm{I}, \mathrm{v} / \mathrm{V}))$ at room temperature as described by Duff et al. (I966). Method (b) was similar to method $(a)$ but with the extraction with each solvent system repeated twice before using the next solvent in the sequence. Method $(c)$ involved sequential Soxhlet extractions (6 hr each) of algal cell powder (100 mg.) with the same solvent systems ( $25 \mathrm{ml}$. samples); each solvent extract was concentrated in vacuo to a $2 \mathrm{ml}$. equivalent of the extracts obtained by the other methods. The ratio of the weight of algal material to final volume of extract used in activity tests was taken as a measure of this equivalence. All extracts were stored at $-30^{\circ}$ and were protected from direct light at all times. 
Activity tests. Seven strains of the test bacteria showing high sensitivity against Isochrysis galbana were chosen from the organisms previously surveyed (Duff et al. I966) with a view to obtaining a fair representation of the activity spectrum; they are listed in Table I. In addition, a freshly isolated organism (Staphylococcus aureus $\mathrm{C}$ from a patient with chronic furunculosis) was included. Activity test methods were as used in the earlier survey. (Duff et al. 1966).

Paper chromatography and bioautography of algal extracts. All solvents (mostly analytical reagent grade) were distilled before use. As far as possible, all operations were done in subdued light or in the dark. A cm. ${ }^{2}$ grid system was marked on a $15 \mathrm{~cm}^{2}$ area of a Whatman no. I chromatography paper sheet $\left(25 \mathrm{~cm} .{ }^{2}\right)$ and a labelling system was devised to enable later identification of each square. A sample $(0.05 \mathrm{ml}$.) of extract was applied to the point of chromatographic origin taken at the centre of a corner square of the grid. Two-dimensional descending chromatography was used with $4 \%(\mathrm{v} / \mathrm{v})$ propan-I-ol in light petroleum (b.p. 60-80 ) as developing solvent in the first dimension and $30 \%(\mathrm{v} / \mathrm{v})$ chloroform in light petroleum (b.p. 60-80 ) in the second dimension. The chromatograms were developed for $\mathrm{I} \mathrm{hr}$ in each dimension, dried and transferred to a sterile container. Test plates were prepared for bioautography by the same methods used earlier for testing extract-charged discs (Duff et al. 1966). Only Brevibacterium sp. C-7b and Micrococcus sp. C-54b were used as test organisms. The developed chromatogram was cut aseptically into $\mathrm{I} \times 5 \mathrm{~cm}$. strips along the lines of the marked grid and placed on the surface of the seeded test plates, which were then incubated and examined as reported by Duff et al. (I966). Areas of inhibition were related to chromatographic $R_{F}$ values from the grid identification marks.

The areas known to be active were cut from subsequent chromatograms and extracted with diethyl ether. These eluates were concentrated to a volume equivalent to that of the original algal extract, for quantitative comparison of activity by disc tests (Table I). Absorption spectra of the eluates were measured on a Beckman DB-G grating spectrophotometer fitted with a linearlog Io in. potentiometric recorder.

\section{RESULTS}

A comparison of the three methods of extraction of the alga showed that exhaustive acetone extraction by either method ( $b$ or $c$ ) was sufficient to remove almost all the active components of the alga and that subsequential extraction with the other solvents was unnecessary. Since the higher temperature $\left(70-75^{\circ}\right)$ of the Soxhlet extraction did not appear to be deleterious to the active components, method (c) was chosen for all subsequent extract preparations because of its convenience.

The bioautography of acetone extracts of the alga showed that all the antibacterial activity was associated with two pigmented components which appeared to be easily separable by adequate development of the chromatogram in the first dimension only. Subsequent unidimensional chromatography of the extracts gave good separation of the slower-moving component $\left(A, R_{F} 0.35\right)$ in about $2.5 \mathrm{hr}$, but required $4 \mathrm{hr}$ for complete separation of the faster-moving component $\left(B, R_{F} 0.9\right)$. Since component $A$ was unstable on prolonged chromatography, it was preferred to run separate chromatograms for isolation of these compounds by elution and to use the minimum period of development required for complete separation of each. 


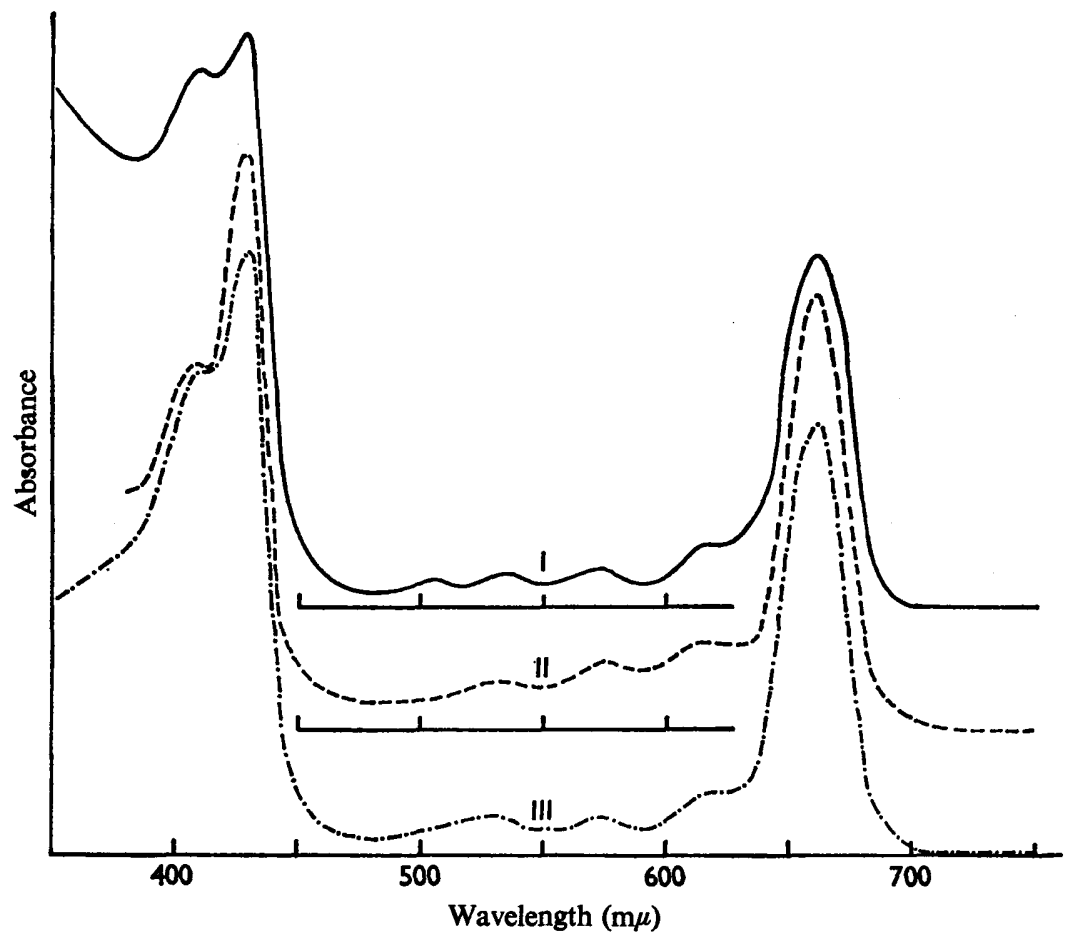

Fig. I. Absorption spectra of compound $A$, ethyl chlorophyllide $a$ (from Holt \& Jacobs, 1954), and atypical chlorophyllide $a$ (from Barrett \& Jeffrey, 1964) in diethyl ether represented by curves I, II, and III respectively.

Table I. Growth inhibition of bacteria from whole extracts of Isochrysis galbana and their active components

Test concentration of the active components was equivalent to that present in the whole extract.

\section{Test bacteria}

Terrestrial strains:

\section{Streptococcus faecalis}

Staphylococcus aureus $\mathrm{C}$

S. aureus $\mathrm{E}$

S. aureus $\mathrm{M}$

Marine strains:

Flavobacterium sp. P-4b Brevibacterium sp. C-7 b

Micrococcus sp. C-54b

Micrococcus sp. P-59

* Prepared by extraction method $c$ (see text).

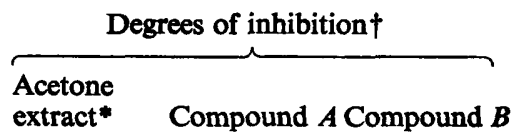

$\dagger$ Growth inhibition was classified into three categories based on diameter of inhibition zones as follows: v, very active $(>1.5 \mathrm{~cm}$.); m, moderately active $(>1 \cdot 0<1.5 \mathrm{~cm}$.); s, slightly active $(<\mathrm{I} \cdot 0 \mathrm{~cm}$.). 
The activities observed from the isolated compounds $A$ and $B$ and from the original acetone extracts are summarized in Table $I$. The results show that little loss or change of activity was incurred during the resolution of extracts and that the isolated compounds possess the same range and similar degrees of activity. Compounds $A$ and $B$ showed blue-green and grey-green hues, respectively, on paper-chromatograms and their locations corresponded closely to those of chlorophyllide $a$ and pheophytin $a$ observed by Jeffrey (I96I) on similarly developed two-dimensional chromatograms of

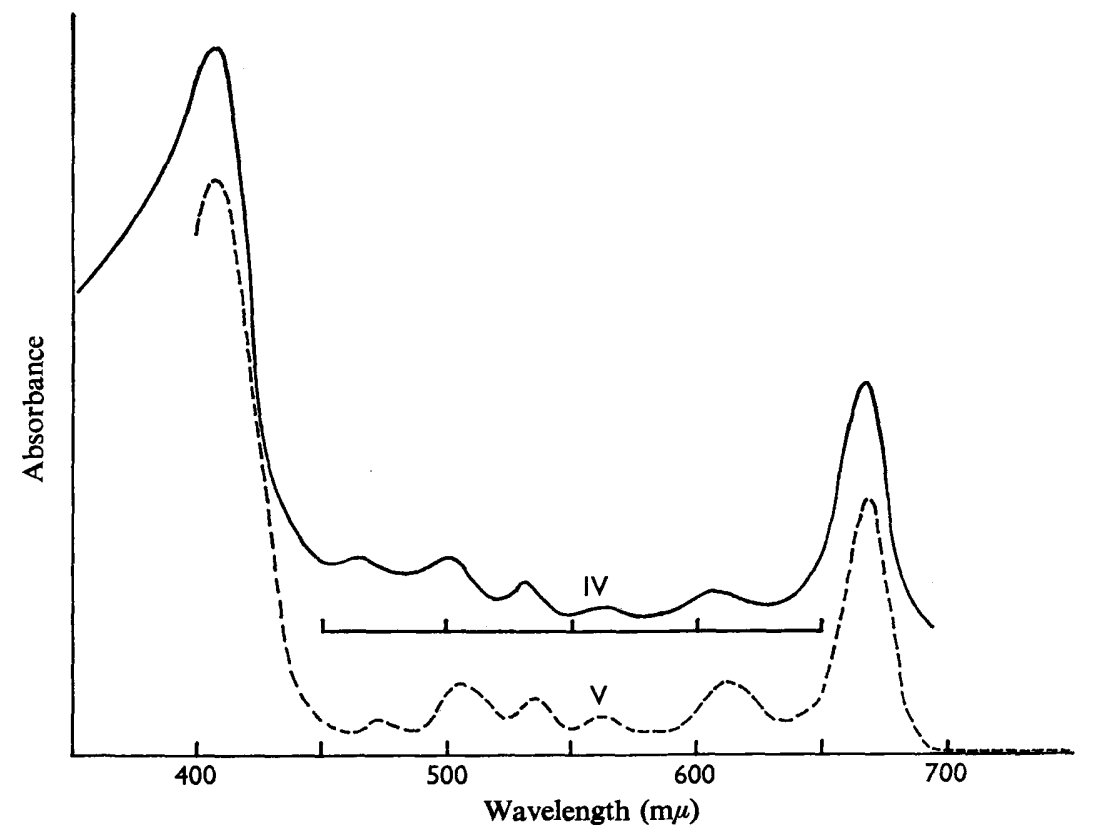

Fig. 2. Absorption spectra of compound $B$ and pheophytin $a$ (from Smith \& Benitez, 1955) in diethyl ether, represented by curves IV and V respectively.

Table 2. Absorption maxima of active components of Isochrysis galbana and some chlorophyll a derivatives

All maxima $(\mathrm{m} \mu)$ are reported for diethyl ether solutions.

\begin{tabular}{llllllll}
\multicolumn{7}{c}{ Absorption maxima (m $\mu$ ) } \\
Compound $A$ & $\overbrace{660}$ & 613 & 573 & 536 & 506 & 428 & 410 \\
Ethyl chlorophyllide $a^{*}$ & $659-660$ & $613-614$ & $574-576$ & $530-532$ & - & $428-429$ & 410 \\
Atypical chlorophyllide $a \dagger$ & 660 & 615 & 573 & 530 & - & 429 & $\sim 415$ \\
Compound $B$ & 668 & 612 & 562 & 536 & 504 & 468 & 408 \\
Pheophytin $a \ddagger$ & 667 & 609.5 & 560 & 534 & 505 & 471 & 408.5
\end{tabular}

* Holt \& Jacobs (1954). † Barrett \& Jeffrey (1964). $\quad \ddagger$ Smith \& Benitez (1955).

marine algal pigments. The first-dimension $R_{F}$ values of 0.38 and 0.87 reported by this author for chlorophyllide $a$ and pheophytin $a$ are also in close agreement with those obtained by us for compounds $A\left(R_{F}, 0.35\right)$ and $B\left(R_{F}, 0.9\right)$. These clues to the identity of the active compounds were verified by their absorption spectra (Figs $I, 2)$. The close correspondence in the absorption of compound $B$ with that previously reported for 
pheophytin $a$ (Fig. 2, Table 2) supports the chromatographic evidence that these two compounds may be identical. The absorption of compound $A$ is very like that of ethyl chlorophyllide $a$ but shows some significant differences (Fig. I and Table 2). Recently, an atypical chlorophyllide $a$ was reported to be produced by some planktonic algae, which differed spectroscopically from the standard chlorophyllide $a$ in the Soret band region and in the ratio of the absorbance of the blue maxima to the red maxima (Barrett \& Jeffrey, 1964). The spectrum of compound $A$ resembles that of the atypical chlorophyllide $a$ in the ratios of the absorbance of the blue maxima ( 428 and 4IO $\mathrm{m} \mu$ ) to the red maximum $(660 \mathrm{~m} \mu)$ but differs in the location of the minor blue maximum (4IO $\mathrm{m} \mu$ ) which is in closer agreement with that of the standard chlorophyllide (see Fig. 2, Table 2). Compound $A$ shows a minor maximum at $506 \mathrm{~m} \mu$ which is absent from the spectra of both chlorophyllides. Although not identical with either chlorophyllide, the overall spectral resemblance and the chromatographic evidence indicate that compound $A$ may be a hitherto uncharacterized chlorophyllide $a$. The recent finding of several 'isomers' of chlorophyll $a$ in Chlorella (Michel-Wolwertz \& Sironval, 1965) anticipates the occurrence of a number of atypical chlorophyllides in algae.

\section{DISCUSSION}

The antibacterial activity of chlorophyll derivatives from algae has been previously reported. Jørgensen (1962) observed growth-inhibition of Bacillus subtilis from photooxidized chlorophyllides and pheophytin-like substances produced by three species of unicellular Chlorophyceae; the active substances were not clearly identified and pheophytin $a$ was stated to be inactive. Sieburth (1965) reported some evidence which suggested that part of the wide-spectrum activity of the diatom Skeletonema costatum might be due to chlorophyll derivatives (presumably a chlorophyllide and its photooxidation product). The present finding of active chlorophyll $a$ derivatives in Isochrysis galbana extends the list of algal classes which produce such antibiotics to the Chrysophyceae and adds weight to the suggestions of Jørgensen (1962) and Sieburth (I965) that active 'chlorophyllides' may play a significant antibacterial role in the microbial ecology of natural waters.

The earlier investigators have generally considered the active 'chlorophyllides' to be degradation products of chlorophyll produced by senescent or autolysed organisms. The culture of Isochrysis galbana used in the present work was harvested towards the end of exponential growth and could not be considered to be senescent, although the possibility that a small proportion of organisms were undergoing premature ageing and autolysis is not excluded. It does seem that bioactive chlorophyll derivatives may also be produced by algae during active growth. That chlorophyllides may not necessarily be products of chlorophyll catabolism is suggested by inspection of the scattered evidence such as the finding of highly active chlorophyllase systems in marine algae (Barrett \& Jeffrey, 1964) and the observation that chlorophyllide $a$ may be an integral part of a major form of in vivo chlorophyll (Sironval, Michel-Wolwertz \& Madsen, I965).

Barrett \& Jeffrey (1964) did not find in Isochrysis galbana the chlorophyllase system which was shown in other algae to produce the atypical chlorophyllide $a$ mentioned earlier (see Results). This confirms our conclusion, based on spectral differences, that the antibacterial chlorophyllide $a$ (compound $A$ ) is not identical with the Barrett-Jeffrey chlorophyllide. Our finding of the antibacterial activity of pheophytin $a$ from I. galbana 
is surprising and requires verification by tests on the compound isolated from other sources, since Jørgensen (1962) found it to be inactive against Bacillus subtilis and yet he obtained pheophytin-like substances from Chlorella, Scenedesmus, and Chlamydomonas which inhibited growth of this bacterium under illumination but not in the dark.

The financial assistance received from the National Research Council of Canada by two of the authors (D. L. B. and D. C. B. D.) in support of this study is gratefully acknowledged.

\section{REFERENCES}

ANTIA, N. J. \& KALMAKOFF, J. (1965). Growth rates and cell yields from axenic mass culture of fourteen species of marine phytoplankters. Fisheries Res. Board Can. Manuscript Rept. Ser. (Oceanog. and Limnol.) no. 203.

BARRETT, J. \& JEFFREY, S. W. (1964). Chlorophyllase and formation of an atypical chlorophyllide in marine algae. Plant Physiol. 39, 44.

DUfF, D. C. B., BRUCE, D. L. \& ANTIA, N. J. (1966). The antibacterial activity of marine planktonic algae. Can. J. Microbiol. 12, 877.

HolT, A. S. \& JACOBS, E. E. (1954). Spectroscopy of plant pigments. I. Ethyl chlorophyllides $a$ and $b$ and their pheophorbides. Am. J. Bot. 4r, 710.

JefrReY, S. W. (1961). Paper-chromatographic separation of chlorophylls and carotenoids from marine algae. Biochem. J. 80, 336.

JøRGENSEN, E. G. (1962). Antibiotic substances from cells and culture solutions of unicellularalgae with special reference to some chlorophyll derivatives. Physiol. Plant. 15, 530.

Michel-WolWERTZ, M.-R. \& Sironval, C. (1965). On the chlorophylls separated by paper chromatography from Chlorella extracts. Biochim. biophys. Acta 94, 330.

SteBURTH, J. MCN. (1965). Role of algae in controlling bacterial populations in estuarine waters. In Pollutions marines par les Microorganismes et les Produits pétroliers, Symposium de Monaco (avril 1964), p. 217. Publ. by Secrétariat général de la Commission Internationale pour l'Exploration scientifique de la Mer Méditerranée, Paris.

Sironval, C., Michel-Wolwertz, M. R. \& MADSEN, A. (1965). On the nature and possible functions of the 673- and 684-m $\mu$ forms in vivo of chlorophyll. Biochim. biophys. Acta 94, 344.

SMITH, J. H. C. \& BenITEZ, A. (1955). Chlorophylls: Analysis in plant materials. In Modern Methods of Plant Analysis. Ed. by K. Paech and M. V. Tracey, 4, 142. Berlin: Springer-Verlag. 